\title{
ARTIGO ORIGINAL \\ Associação entre tuberculose e consumo de drogas lícitas e ilícitas
}

Mariel Tobal Justo*, Luciano Garcia Lourenção, D.Sc. ${ }^{*}$, Natália Sperli Geraldes Marin dos Santos Sasaki, D.Sc. ${ }^{* \star *}$, Silvia Helena Figueiredo Vendramini, D.Sc. ${ }^{* \star *}$, Nilza Gomes de Souza ${ }^{\star \star * *}$, Maria de Lourdes Sperli Geraldes Santos, M.Sc. ${ }^{* \star *}$

\begin{abstract}
*Enfermeira, Mestranda em Enfermagem pela Faculdade de Medicina de São José do Rio Preto (FAMERP), ${ }^{* *}$ Enfermeiro, Professor Titular-Livre da Escola de Enfermagem da Universidade Federal do Rio Grande (EEnf/FURG), ${ }^{* \star}$ Enfermeira, Professora do Curso de Medicina da União das Faculdades dos Grandes Lagos (UNILAGO), ${ }^{* * *}$ Enfermeira, Professora Adjunta do Departamento de Enfermagem em Saúde Coletiva e Orientação Profissional da Faculdade de Medicina de São José do Rio Preto (FAMERP), ${ }^{* \star * \star}$ Enfermeira, Enfermeira da Secretaria de Estado da Saúde de São Paulo e Professora do Centro Estadual de Educação Tecnológica Paula Souza de São José do Rio Preto
\end{abstract}

Recebido em 9 de novembro de 2017; aceito em 5 de junho de 2018.

Endereço para correspondência: Mariel Tobal Justo, Avenida Bady Bassitt, 4370/13, Torre 4, Nossa Senhora Aparecida, 15025-000 São José do Rio Preto SP, E-mail: mariel_famerp@yahoo.com.br; Luciano Garcia Lourenção: luciano.famerp@gmail.com; Natália Sperli Geraldes Marin dos Santos Sasaki: nsperli@gmail.com; Silvia Helena Figueiredo Vendramini: silviahve@gmail.com; Nilza Gomes de Souza: gve29-tbmonitor@saude.sp.gov.br; Maria de Lourdes Sperli Geraldes Santos: mlsperli@gmail.com

\section{Resumo}

Estudo transversal sobre a associação entre características clínicas e sociodemográficas, descoberta e desfecho de tratamento da tuberculose com uso de drogas lícitas e ilícitas. Foram utilizados dados secundários dos casos de tuberculose notificados no sistema TB-WEB, no período de 1997 a 2015, pelos municípios pertencentes ao Grupo de Vigilância Epidemiológica 29, de São José do Rio Preto, São Paulo. Realizou-se análise de frequência simples e associativa, aplicando-se os testes qui-quadrado e Exato de Fisher, considerando nível de significância de $95 \%$. Os resultados mostraram predomínio de pacientes usuários de álcool, outras drogas e tabaco, sexo masculino, faixa etária de 20 a 59 anos, brancos, com 4 a 7 anos de escolaridade, notificados como casos novos de tuberculose pulmonar. O diagnóstico ocorreu em nível ambulatorial e hospitalar, com índices de cura inferiores à meta do Ministério da Saúde. Os resultados apontaram associação significativa entre doentes de tuberculose e uso de álcool, outras drogas e tabaco, exigindo especial atenção para o enfrentamento do problema. Estratégias devem ser desenvolvidas para uma reorganização dos serviços de saúde, além do olhar atento para esses pacientes vulneráveis.

Palavras-chave: tuberculose, drogas ilícitas, alcoolismo, tabaco, tratamento.

\begin{abstract}
Association between tuberculosis and use of licit and illicit drugs

Cross-sectional study about association between clinical and socio-demographic characteristics, discovery and treatment outcome of tuberculosis with licit and illicit drugs use. Secondary data were used in cases of tuberculosis notified in TB-WEB system in the period of 1997 to 2015, in municipalities belonging to Group of Epidemiological Surveillance 29 of São José do Rio Preto, São Paulo state. A simple and associative frequency analysis was performed through the chisquare test and Fisher's exact test, considering a significance level of $95 \%$. The results showed predominance of patients users of alcohol, other drugs and tobacco, male, aged 20 to 59 years old, white, with 4 to 7 years of schooling, notified as new cases of pulmonary tuberculosis. The diagnosis occurred in outpatient and hospital level, with cure rates lower than the objective of the Health Ministry. The results showed a significant association between tuberculosis patients and use of alcohol, other drugs and tobacco, requiring special attention for confrontation of the problem. Strategies should be developed for a reorganization of health services, in addition to look out for these vulnerable patients.
\end{abstract}

Key-words: tuberculosis, illicit drugs, alcoholism, tobacco, treatment. 


\section{Resumen \\ Asociación entre tuberculosis y el consumo de drogas lícitas e ilícitas}

Estudio transversal sobre la asociación entre características clínicas y sociodemográficas, descubrimiento y desenlace del tratamiento de la tuberculosis con el uso de drogas lícitas e ilícitas. Se utilizaron datos secundarios de los casos de tuberculosis notificados en el sistema TB-WEB, en el período de 1997 a 2015, por los municipios pertenecientes al Grupo de Vigilancia Epidemiológica 29, de São José do Rio Preto, São Paulo. Se realizó un análisis de frecuencia simple y asociativa, aplicando las pruebas qui-cuadrado y Exacto de Fisher, considerando nivel de significancia de $95 \%$. Los resultados mostraron predominio de pacientes usuarios de alcohol, otras drogas y tabaco, sexo masculino, grupo de edad de 20 a 59 años, blancos, con 4 a 7 años de escolaridad, notificados como casos nuevos de tuberculosis pulmonar. El diagnóstico ocurrió a nivel ambulatorio y hospitalario, con índices de curación inferiores a la meta del Ministerio de Salud. Los resultados apuntaron una asociación significativa entre enfermos de tuberculosis y uso de alcohol, otras drogas y tabaco, exigiendo especial atención para el enfrentamiento del problema. Las estrategias deben ser desarrolladas para una reorganización de los servicios de salud, además de la mirada atenta a esos pacientes vulnerables.

Palabras-clave: tuberculosis, drogas ilícitas, alcoholismo, tabaco, tratamiento.

Introdução

A tuberculose (TB) tem ganhado notoriedade, por se tratar de um problema global de saúde pública, devido à sua elevada prevalência em diversos países, dentre eles o Brasil. A doença tem estreita relação com condições socioeconômicas desfavoráveis e permanece como uma das doenças infecciosas que causa o maior número de óbitos em adultos [1-2].

É causada pelo Mycobacterium tuberculosis, bactéria também denominada Bacilo do Koch, e a principal fonte de infecção é a forma pulmonar. A TB é curável em $100 \%$ dos casos, desde que o paciente busque o tratamento, que é gratuito na rede pública de saúde. Apesar disso, a doença ainda leva a óbito cerca de 4,5 mil pessoas por ano no Brasil [3-4]. Estima-se que um terço da população mundial esteja infectada com o bacilo causador da doença e que, em 2013, ocorreram 9 milhões de casos novos e 1 milhão de óbitos. A cada ano, são notificados aproximadamente 70 mil casos novos e ocorrem 4,5 mil mortes em decorrência da doença [5].

A nova classificação para o controle da TB em países prioritários, para o período de 2016 a 2020, é composta por três listas de 30 países, que concentram $87 \%$ do número de casos de tuberculose no mundo. A nova classificação se baseia nas seguintes características epidemiológicas: carga de tuberculose, tuberculose multidrogarresistente e coinfecção TB/HIV [5].

O Brasil encontra-se em duas destas classificações e ocupa a 20 a posição em carga de TB e a 19a quanto à coinfecção TB/HIV, concentrando $0,9 \%$ dos casos estimados no mundo $e$ $33 \%$ dos estimados para as Américas. Embora o país tenha cumprido as metas internacionais, com redução dos coeficientes de mortalidade e de incidência da doença, de 1990 a 2014, em $38,9 \%$ (3,6 para 2,2/100 mil habitantes) e $34,1 \%$ (51,8 para $34,1 / 100$ mil habitantes), respectivamente [5], entre 2005 e 2014, houve uma média de 70 mil casos novos e 4.400 mortes anuais por tuberculose no país; e entre 2012 e 2015, 840 casos novos de tuberculose drogarresistente [6].

Apesar de ser uma doença curável, de fácil diagnóstico e tratamento efetivo, fatores como o abandono do tratamento dificultam o controle da TB, em todo o mundo [5,7-9]. Além disso, fatores como a infecção por HIV, pessoas que fazem uso frequente de drogas lícitas e ilícitas, e o baixo padrão socioeconômico favorecem a transmissão da TB pulmonar [10].

No Brasil, o Ministério da Saúde preconiza uma taxa de abandono inferior a 5\%, no entanto, em 2011, 8,9\% dos doentes diagnosticados no país abandonaram o tratamento [7-9].

Nos últimos anos, a piora das condições socioeconômicas no Brasil causou impacto negativo no controle da TB, devido à relação da doença com baixa renda familiar, educação precária, habitação fora dos padrões sanitários, alimentação inadequada, coinfecção HIV/AIDS e uso do tabaco, álcool e outras drogas [7,11].

De acordo com a literatura, há forte relação entre TB e consumo frequente de drogas ilícitas, álcool e tabaco, sendo este último mais expressivo, por conta da presença da nicotina, que se revelou importante na reativação da TB em casos considerados encerrados, pois 
diminui a resistência do hospedeiro e promove alterações alveolares, aumentando o risco de persistência da bactéria após o tratamento [12,14].

Segundo a Organização Mundial de Saúde, o tabagismo aumenta em mais de 2,5 vezes o risco de adquirir tuberculose e, consequentemente, morrer devido à doença [13]. Além disso, o uso de drogas ilícitas é um dos principais responsáveis pelo abandono do tratamento de TB, potencializado por aspectos sociodemográficos, relacionados aos serviços de saúde e ao tratamento da doença, comorbidades e o cuidado com a saúde em geral [14].

Estudo sobre a correlação entre tabagismo e os desfechos do tratamento da TB identificou que, além de o tabagismo prejudicar a eficácia do tratamento de fumantes, afeta negativamente $o$ tratamento de ex-fumantes [15]. Esses resultados evidenciam a necessidade de restrições específicas e ações de redução das taxas de utilização do tabaco pelos doentes de TB, para melhorar a eficácia do tratamento e a qualidade dos serviços de saúde prestados a esta população.

Destaca-se, ainda, a associação do consumo excessivo de álcool com o aumento do risco de infecção pela TB pulmonar e maior risco de intoxicação por drogas. O alcoolismo também está relacionado a outros determinantes relevantes, como baixo status socioeconômico, desnutrição e ausência de moradia fixa [16].

Ante o exposto, este estudo objetivou verificar a associação entre o uso de drogas ilícitas, álcool e tabaco, e as características sociodemográficas, clínicas, descoberta e desfecho de tratamento dos casos de tuberculose notificados ao Grupo de Vigilância Epidemiológica XXIX (GVE-29) de São José do Rio Preto, no período de 1997 a 2015.

\section{Material e métodos}

Estudo transversal sobre a associação entre características clínicas e sociodemográficas, descoberta e desfecho de tratamento da TB com uso de drogas ilícitas, álcool e tabaco. Foram utilizados dados secundários dos casos de tuberculose notificados no sistema TB-WEB, no período de 1997 a 2015, pelos municípios pertencentes ao Grupo de Vigilância Epidemiológica 29 (GVE 29), de São José do Rio Preto, São Paulo. O GVE 29 é um dos 32 grupos integrantes, em nível regional, da estrutura do Centro de Vigilância Epidemiológica "Prof. Alexandre Vranjac" (CVE), que normatiza o Sistema de Vigilância Epidemiológica no Estado de São Paulo. Abrange 67 municípios pertencentes aos Colegiados de Gestão Regionais de Catanduva, José Bonifácio, Votuporanga e São José do Rio Preto, e está inserido na divisão administrativa da Regional de Saúde XV, uma das maiores regionais do Estado de São Paulo [17].

Foram incluídos no estudo os casos de TB em usuários de drogas ilícitas, alcoolistas e tabagistas, independentemente da idade ou sexo, notificados no período de 1997 a 2015 no sistema de informação TB-WEB, residentes nos municípios da área de atuação do GVE 29 de São José do Rio Preto. Excluíram-se os doentes de TB usuários de drogas ilícitas, alcoolistas e/ou tabagistas transferidos para outro Estado ou GVE, com tratamento sem encerramento e mudança de diagnóstico.

As variáveis escolhidas para a análise foram estratificadas em Demográficas: sexo (feminino/masculino), faixa etária (até 19 anos, 20 a 59 anos, 60 ou mais e sem informação), raça/cor, escolaridade ( 0 , de 1 a 3 anos, 4 a 7 anos, 8 a 11 anos, 12 ou mais e sem informação); Município notificante (São José do Rio Preto e região); e Clínicas: tipo de caso (novo ou recidiva), classificação, encerramento e descoberta.

Foi considerado caso novo o paciente que nunca usou medicamentos antituberculose, ou usou por menos de 30 dias [18].

Foram realizadas análises de frequência simples e associativa, aplicando-se os testes qui-quadrado e Exato de Fisher, considerando nível de significância de 95\%.

O estudo foi aprovado pelo Comitê de Ética em Pesquisa da Faculdade de Medicina de São José do Rio Preto (FAMERP) em 22 de abril de 2015, com Parecer №. 1.030.405.

Foram avaliados 879 casos de TB, notificados no período do estudo. Conforme observado na Tabela I, houve prevalência da raça/cor branca $(58,5 \%)$ e parda $(21,4 \%)$; faixa etária produtiva - 20 a 59 anos $(58,5 \%)$; sexo masculino $(84,4 \%)$. A escolaridade mostrou-se baixa, 536 doentes $(61,0 \%)$ tinham de 0 a 7 anos de estudo, equivalente ao ensino 
fundamental incompleto. A maioria das notificações foi realizada pelo município de São José do Rio Preto $(58,4 \%)$.

Tabela I - Distribuição dos casos de tuberculose notificados ao GVE 29 no período de 1997 a 2015, segundo variáveis demográficas, escolaridade e município notificante.

\begin{tabular}{lcc}
\hline \multicolumn{1}{c}{ Variáveis } & $\mathbf{n}$ & $\%$ \\
\hline Sexo & 752 & 85,6 \\
Masculino & 127 & 14,4 \\
Feminino & 13 & 1,5 \\
\hline Faixa etária (anos) & 742 & 84,4 \\
Até 19 & 124 & 14,1 \\
20 a 59 & & \\
60 ou mais & 514 & 58,5 \\
\hline Raça/cor & 188 & 21,4 \\
Branco & 79 & 9,0 \\
Pardo & 6 & 0,7 \\
Preto & 92 & 10,4 \\
Outro & 34 & 3,9 \\
Sem informação & 132 & 15,0 \\
Escolaridade (anos) & 370 & 42,1 \\
0 & 230 & 26,2 \\
1 a 3 & 22 & 2,5 \\
4 a 7 & 91 & 10,3 \\
8 a 11 & & \\
12 ou mais & 513 & 58,4 \\
Sem Informação & 366 & 41,6 \\
\hline Município notificante &
\end{tabular}

Em relação às variáveis clínicas e de descoberta da doença (Tabela II), observou-se maior porcentual de casos novos $(90,1 \%)$ e cura $(74,1 \%)$, além de $9,7 \%$ de abandono e $15,4 \%$ de óbitos; prevalência da forma pulmonar (86,5\%). Em relação ao diagnóstico da TB, destacase o baixo índice de descoberta por busca ativa de sintomáticos respiratórios $(7,0 \%)$ e as elevadas taxas de diagnóstico por demanda ambulatorial $(37,8 \%)$ e fora dos serviços de Atenção Básica, ou seja, durante internação hospitalar (30,6\%), em Unidades de Pronto Atendimento $(21,8 \%)$ e após o óbito $(2,2 \%)$.

Entre os casos TB estudados, $254(29,6 \%)$ eram usuários de drogas ilícitas, 410 $(47,7 \%)$ tabagistas, e 500 (58,2\%) alcoolistas. Houve associação do uso de drogas ilícitas, álcool e tabaco com as variáveis demográficas, sociais e município notificante, exceto alcoolismo e município notificante (Tabela III). Maior consumo de tabaco e drogas ilícitas entre mulheres - $63,0 \%$ fumantes e $43,3 \%$ usuárias de drogas ilícitas. Entre os homens houve maior consumo de álcool $(68,8 \%)$.

O consumo de tabaco e álcool foi maior entre doentes com idade entre 20 e 59 anos (47,7\% e $66,4 \%$ respectivamente) e com mais de 60 anos (67,7\% tabaco e $64,8 \%$ álcool); o uso de drogas ilícitas predominou entre os adolescentes (61,5\%). Ressalta-se, ainda, que o alcoolismo foi maior que os outros eventos em todas as faixas etárias.

Houve prevalência do consumo de álcool entre os negros $(70,9 \%)$ e associação da escolaridade com uso de drogas ilícitas, tabagismo e alcoolismo. O maior consumo de tabaco ocorreu entre doentes com maior escolaridade $(59,1 \%)$, enquanto o consumo de álcool foi maior entre os de menor escolaridade - $68,9 \%$ para 1 a 3 anos de estudo e $67,6 \%$ para os sem escolaridade. 
Tabela II - Distribuição dos casos de tuberculose notificados ao GVE 29 no período de 1997 a 2015, segundo variáveis clínicas e de descoberta.

\begin{tabular}{lcc}
\multicolumn{1}{c}{ Variáveis } & $\mathbf{n}$ & $\%$ \\
\hline Tipo de caso & 787 & $90,1 \%$ \\
Novo & 92 & $9,9 \%$ \\
Recidiva & & \\
Encerramento & 651 & 74,1 \\
Cura & 85 & 9,7 \\
Abandono & 102 & 11,6 \\
Óbito Não TB & 33 & 3,7 \\
Óbito TB & 8 & 0,9 \\
Sem informação & & \\
\hline Classificação & 732 & 83,3 \\
Pulmonar & 115 & 13,1 \\
Extrapulmonar & 28 & 3,2 \\
Pulmonar/Extrapulmonar & 4 & 0,4 \\
Disseminada & & \\
\hline Descoberta & 62 & 7,0 \\
Busca ativa & 332 & 37,8 \\
Demanda ambulatorial & 19 & 2,2 \\
Descoberta após óbito & 269 & 30,6 \\
Internação & 192 & 21,8 \\
Urgência / Emergência & 5 & 0,6 \\
Sem informação & &
\end{tabular}

Conforme observado na Tabela IV, não houve associação do consumo de tabaco com classificação da doença $(p=0,90)$ e descoberta $(p=0,06)$; uso de drogas ilícitas com classificação da doença $(p=0,15)$; alcoolismo com encerramento $(p=0,14)$ e classificação da doença $(p=0,83)$.

Houve maior recidiva da TB entre alcoolistas $(72,8 \%)$. Entre os curados, observou-se maior prevalência de alcoolistas $(62,2 \%)$ e menor de uso de drogas ilícitas $(30,9 \%)$. No entanto, alcoolistas $(69,4 \%)$ e usuários de drogas ilícitas $(64,7 \%)$ abandonaram o tratamento mais do que tabagistas (44,7\%). Houve maior ocorrência de óbito entre fumantes $(63,6 \%$ por TB e $57,8 \%$ não TB) e alcoolistas (71,6\% não TB e por TB e 69,7\%). alcoolistas.

Entre todas as formas de descoberta da doença houve predomínio de tabagistas e

Tabela III - Associação do uso de drogas ilícitas, álcool e tabaco com variáveis demográficas, escolaridade e município notificante, entre doente de TB notificados ao GVE 29 no período de 1997 a 2015. (ver anexo em PDF).

Tabela IV - Associação do uso de drogas ilícitas, álcool e tabaco com variáveis clínicas e de descoberta entre doente de TB notificados ao GVE 29 no período de 1997 a 2015. (ver anexo em PDF).

Discussão

O estudo mostrou prevalência de doentes do sexo masculino, na faixa etária produtiva, da raça/cor branca e parda, com baixa escolaridade, TB pulmonar, notificados pelo município de São José do Rio Preto, com diagnóstico fora dos serviços de Atenção Básica e evolução para cura.

A prevalência de casos de TB entre homens é comum em todas as faixas etárias. Isso ocorre devido à maior exposição dos homens a fatores de risco para a TB, ou em virtude das diferenças culturais no desempenho de papéis entre os sexos, que engloba, entre outros, a procura limitada pelos serviços de saúde por parte dos homens, além do estilo de vida de cada indivíduo [19]. O predomínio da raça/cor branca condiz com a distribuição da população do estado de São Paulo composta, majoritariamente, por pessoas raça/cor branca [20]. 
Assim como em outros estudos epidemiológicos, predominou o baixo nível de escolaridade [21-23]. Esta condição exige maior atenção dos profissionais da saúde com o doente de TB em tratamento, pois a baixa escolaridade é um indicador de vulnerabilidade social, que contribui para o retardo do diagnóstico, não aceitação da doença e baixa adesão ao tratamento [7,24-25].

A TB pulmonar, predominante neste estudo, é a mais comum e de maior relevância para a saúde pública, especialmente a pulmonar bacilífera, responsável pela manutenção da cadeia de transmissão da doença [3]. Como a doença é transmitida por gotículas expelidas pelos doentes, através da fala, do espirro e da tosse, na TB pulmonar as gotículas mais leves podem permanecer em suspensão no ar, por diversas horas [26].

Este estudo apontou, ainda, associação entre uso de drogas ilícitas, álcool e tabaco com variáveis demográficas, escolaridade e município notificante, exceto para alcoolismo e município notificante; ausência de associação do tabagismo com classificação da doença e descoberta, do uso de drogas ilícitas com classificação da doença e do alcoolismo com classificação da doença e encerramento.

Enquanto o predomínio do consumo de álcool por doentes do sexo masculino apresenta um aspecto cultural, associado a questões de masculinidade e poder [25,27-28], o maior consumo de tabaco e drogas ilícitas entre as mulheres com TB é preocupante, pois estas são mais vulneráveis aos danos causados pelas drogas e, certamente, sofrerão maiores impactos ao longo do tratamento [24].

O maior consumo de tabaco e álcool por doentes em idade produtiva está relacionado à distribuição etária da população brasileira e corrobora a literatura [19,22-23,28]. Por outro lado, enquanto este estudo mostrou associação entre uso de drogas ilícitas e tabaco por doentes da raça/cor branca, a literatura aponta que o risco de morrer por tuberculose é maior entre negros e pardos [19,22-24].

O consumo de drogas ilícitas, álcool e tabaco associado à TB representa um grande desafio para a saúde global, pois cria uma vulnerabilidade social que dificulta o controle da doença. Além de a interação nociva destas drogas com o organismo do doente comprometer sua capacidade de compreensão sobre a importância do tratamento, e dificultar a atuação dos profissionais de saúde [29], geralmente a abordagem deste problema ocorre de forma fragmentada, por meio de ações e programas coordenados por infectologistas, psiquiatras e pneumologistas, ao invés de uma abordagem integrada [9,30-31].

O alcoolismo torna-se um importante complicador ao dificultar a adesão do doente ao tratamento e/ou inibir os efeitos da medicação. Além de ser comum o doente optar pela bebida, o uso concomitante de medicação e álcool inibe os efeitos dos remédios, comprometendo o tratamento e aumentando o risco de intolerância à medicação [9,29,32-35]. Em pacientes de retratamento, a não-aderência ao uso dos medicamentos frequentemente está associada ao consumo perigoso de álcool, confirmando a influência negativa da droga no tratamento da doença [11].

Em relação às drogas ilícitas, o crack se destaca por ser uma droga ampla e rapidamente difundida, de baixo custo, que atinge todas as classes sociais e faixas etárias. Há uma íntima relação entre a pobreza e a vulnerabilidade ao uso de crack, por seu menor custo e efeito quase instantâneo. Sua relação com a TB se deve ao fato de o baixo poder aquisitivo privar as pessoas de condições dignas de habitação e de vida, levando-as a viver em áreas de infraestrutura urbana precária, aglomeradas em pequenos espaços, tornando-se mais vulneráveis à contaminação. Além disso, a redução dos níveis de consciência causada pelo uso desta droga, aliada aos efeitos colaterais da medicação antituberculosa, dificultam o tratamento dos doentes de TB usuários de crack $[9,31,36]$.

De acordo com a literatura, indivíduos que fazem uso de drogas ilícitas são três vezes mais propensos à infecção por TB do que aqueles que não consomem substâncias tóxicas. Isto ocorre devido à redução da imunidade causada por estas drogas, associada ao maior risco de disseminação do bacilo nos ambientes de consumo, conhecidos como mocós, geralmente pequenos, superlotados e com pouca circulação de ar [10].

Ademais, os usuários de drogas ilícitas apresentam dificuldade de reconhecer os sintomas tradicionais da doença, como tosse e expectoração; mesmo após o diagnóstico, apresentam-se tardiamente nos serviços de saúde, para iniciar o tratamento e têm a tendência de não aderir ao tratamento e esconder a doença de seus pares, por medo de serem abandonados e proibidos de compartilhar drogas [37].

As drogas ilícitas representam, portanto, um grande obstáculo para o controle da TB, pois os usuários apresentam comportamento vulnerável, não fazem uso correto da medicação, não 
se alimentam bem e não seguem as orientações do tratamento proposto, aumentando o risco de insucesso do tratamento e favorecendo a retransmissão do bacilo, em nível familiar, social e comunitário [14].

Além do alcoolismo e das drogas ilícitas, o tabagismo também ocupa espaço importante na manutenção da cadeia de transmissão da TB, assim como na sua potencialização. Fumar aumenta o risco da tuberculose de infecção latente (TBIL), da progressão da TB ativa, de atraso e negativação do exame de escarro, reduz a adesão ao tratamento, aumenta a recidiva e contribui para a multirresistência $[30,38]$. Os fumantes apresentam risco 2,5 vezes maior de TB recorrente, comparados aos não fumantes, além de apresentarem menor adesão ao tratamento [30]. Ainda, a inalação da fumaça de forma passiva, pode aumentar o risco de TB intra-domiciliar [38].

Não obstante, ao favorecer o abandono do tratamento, o uso descontrolado de tabaco e álcool, e o consumo de drogas ilícitas pelos doentes de TB aumenta o risco de mortalidade pela doença $[9,14,39-40]$.

Além disso, os altos índices de diagnóstico destes doentes em serviços ambulatoriais e de internação, exige maior integração das unidades da atenção básica, em particular a Estratégia Saúde da Família, com as ações de vigilância em saúde, a fim de potencializar as ações de controle da TB, como aumento da busca ativa de sintomáticos respiratórios, redução dos casos de abandono de tratamento e aumento dos índices de cura.

Considerando a vulnerabilidade que permeia a relação TB versus consumo de drogas lícitas e ilícitas, é imprescindível que os profissionais dos serviços de atenção básica identifiquem doentes nestas condições, orientando-os e implementando medidas que busquem a redução do consumo de drogas, para potencializar a eficácia do tratamento. A comunicação entre profissional de saúde e doente é fundamental para a criação de vínculo e o estabelecimento de um relacionamento de confiança, que permita o bom andamento do tratamento, especialmente quando se trata de usuários de drogas ilícitas [19,21-23,29].

Os resultados obtidos neste estudo são relevantes e robustos. No entanto, lacunas no preenchimento das variáveis nas fichas de notificação dificultaram a obtenção dos dados, representando uma limitação do estudo. Nesse contexto, destaca-se a importância da implementação de ações educativas para orientação e capacitação dos profissionais sobre o preenchimento adequado das fichas de notificação, para assegurar a qualidade dos dados e a utilização dos sistemas de informação como fonte para a análise de situação de saúde da população.

Os resultados deste estudo mostraram uma associação significativa entre a TB e 0 consumo de álcool, outras drogas e tabaco, reforçando a tuberculose como um importante problema de saúde pública, agravado pelo consumo destas drogas lícitas e ilícitas pelos doentes.

Estes resultados evidenciam a necessidade de implementação de estratégias de reorganização dos serviços de saúde, além de um olhar atento dos profissionais para esses pacientes vulneráveis, com o objetivo de reduzir os impactos negativos que o consumo destas drogas causa ao tratamento.

A atuação dos profissionais de saúde, por meio do acolhimento e do vínculo com os pacientes é fundamental para aumentar a eficácia e reduzir o abandono do tratamento.

Referências

1. Alcântara CCS, Kritski AL, Ferreira VG, Façanha MC, Pontes RS, Mota RS, et al. Fatores associados à tuberculose pulmonar em pacientes que procuraram serviços de saúde de referência para tuberculose. J Bras Pneumol 2012;38(5):622-9.

2. Alves S, Souza KMJ, Oliveira AAV, Palha PF, Nogueira JA, Sá LD. Tuberculosis treatment abandonment and comprehensive health care to patients in the family healthcare strategy. Texto Contexto Enferm 2012;21(3):650-7.

3. Brasil. Ministério da Saúde. Secretaria de Vigilância em Saúde. Departamento de Vigilância Epidemiológica. Manual de recomendações para o controle da tuberculose no Brasil. Brasília: Ministério da Saúde; 2011. [citado 2017 Nov7]. Disponível em: http://bvsms.saude.gov.br/bvs/publicacoes/manual_recomendacoes_controle_tuberculo se_brasil.pdf. 
4. Augusto CJ, Carvalho WS, Gonçalves AD, Ceccato MGB, Miranda SS. Características da tuberculose no estado de Minas Gerais entre 2002 e 2009. J Bras Pneumol 2013;39(3):357-64.

5. World Health Organization. Global tuberculosis report 2015. 20 ed. Geneva, Switzerland; 2015. 115p.

6. Sharma SK, Vashishtha R, Chauhan LS, Sreenivas V, Seth D. Comparison of TST and IGRA in diagnosis of latent tuberculosis infection in a high TB-burden setting. PLoS One 2017;12(1):1-11.

7. Araujo AS, Vieira SS, Lucena Junior B. Fatores condicionantes ao abandono do tratamento da tuberculose relacionados ao usuário e à equipe de saúde. Cad Saúde Desenvol 2017;10(6):18-23.

8. Rodrigues ILA, Monteiro LL, Pacheco RHB, Silva SED. Abandono do tratamento de tuberculose em co-infectados TB/HIV. Rev Esc Enferm USP 2010;44(2):383-7.

9. Couto DS, Carvalho RN, Azevedo EB, Moraes MN, Pinheiro PGOD, Faustino EB. Fatores determinantes para o abandono do tratamento da tuberculose: representações dos usuários de um hospital público. Saúde Debate 2014;38(102):572-81.

10. Al-Darraji HAA, Wong KC, Yeow DGE, Fu JJ, Loeliger K,Paiji C et al. Tuberculosis screening in a novel substance abuse treatment center in Malaysia: implications for a comprehensive approach for integrated care. J Subst Abuse Treat 2014;46(2):144-9.

11. Peltzer K, Louw J, Mchunu G, Naidoo P, Matseke G, Tutshana B. Hazardous and harmful alcohol use and associated factors in tuberculosis public primary care patients in South Africa. Int J Environ Res Public Health 2012;9(9):3245-57.

12. Rodrigues IC, Helena S, Vendramini F, Amélia M, Ponce Z, Ruffino-Neto A et al. Recidiva da Tuberculose?: fatores associados em um Grupo de Vigilância Epidemiológica de São Paulo. Rev Eletr Enf 2017;19:a06. http://dx.doi.org/10.5216/ree.v19.42691.

13. World Health Organization. A WHO / The Union monograph on TB and tobacco control: joining efforts to control two related global epidemics. Geneva: WHO; 2007. 88p.

14. Cassiano JGM. Tuberculose pulmonar e o uso de drogas ilícitas: entre a cura e o abandono [Dissertação]. Fortaleza: Universidade Federal do Ceará; Programa de PósGraduação em Enfermagem; 2014.

15. Balian DR, Davtyan K, Balian A, Grigoryan A, Hayrapetyan A, Davtyan H. Tuberculosis treatment and Smoking, Armenia, 2014-2016. J Clin Tuberc Other Mycobact Dis 2017;8:1-5.

16. Silva VD, Mello FCQ, Figueiredo SCA. Estimativas das taxas de recidiva, cura e abandono de tratamento em pacientes com tuberculose pulmonar tratados com esquema de dose fixa combinada de quatro drogas em uma unidade de saúde terciária na cidade do Rio de Janeiro. J Bras Pneumol 2017;43(2):113-20.

17. Brasil. Ministério da Saúde. Secretaria de Vigilância em Saúde. Departamento de Vigilância Epidemiológica. Manual de Recomendações para o Controle da Tuberculose no Brasil. Brasília: Ministério da Saúde; 2011.

18. São Paulo. Secretaria de Estado da Saúde. Centro de Vigilância Epidemiológica "Prof. Alexandre Vranjac". Relação dos grupos e subgrupos de Vigilância Epidemiológica (2017). [citado 2017 Nov 7]. Disponível em: http://www.cve.saude.sp.gov.br/htm/outros/ gves.html.

19. Fiori NS, Faria NMX, Meucci RD, Fassa AG. Prevalência e fatores associados ao tabagismo em fumicultores do Sul do Brasil. Cad Saúde Pública 2016;32(7):e00123115.

20. Brasil. Instituto Brasileiro de Geografia e Estatística (IBGE). Censo 2010. [citado 2017 Nov 7]. Disponível em: https://censo2010.ibge.gov.br/resultados.html.

21. Malta DC, Oliveira TP, Luz M, Stopa SR, Silva Junior JB, Reis AAC. Smoking trend indicators in Brazilian capitals, 2006-2013. Cienc Saude Coletiva 2015;20(3):631-40.

22. Araújo DS, Santos FC, Marinho JR, Corrêa SA, Carvalho TLS. Associação entre tuberculose e uso de drogas psicotrópicas em pacientes internados em um hospital público de referência na cidade de Salvador/BA. Fisioter Bras 2015;16(2):113-7.

23. Botti NCL, Machado JSA, Tameirao FV. Perfil sociodemográfico e padrão do uso de crack entre usuários em tratamento no Centro de Atenção Psicossocial. Estud Pesqui Psicol 2014;14(1):290-303.

24. Giroti SKO, Belei RA, Moreno FN, Silva FS. Perfl dos pacientes com tuberculose e os fatores associados ao abandono do tratamento. Cogitare Enferm 2010;15(2):271-7. 
25. Malta DC, Iser BPM, Sá NNB, Yokota RTC, Moura L, Claro RM et al. Tendências temporais no consumo de tabaco nas capitais brasileiras, segundo dados do VIGITEL, 2006 a 2011. Cad Saúde Pública 2013;29(4):812-22.

26. Bertolozzi MR, Takahashii RF, Hino P, Litvoc M, França FOS. O controle da tuberculose um desafio para a saúde pública. Rev Med (São Paulo) 2014;93(2):83-9.

27. Andrade AT, Rimes TS, Costa LSP, Jorge MSB, Quinderé PHD. Aspectos sociodemográficos dos usuários de crack assistidos pela rede de atenção psicossocial. SMAD, Rev Eletrônica Saúde Mental Álcool Drog 2016;12(1):40-7.

28. Garcia LP, Freitas LRS. Consumo abusivo de álcool no Brasil: resultados da Pesquisa Nacional de Saúde 2013. Epidemiol Serv Saúde 2015;24(2):227-37.

29. Souza LO, Mitano F, Lima MCRA d' A, Sicsú AN, Silva LMCa, Palha PF. Terapia de curta duração da tuberculose: uma análise discursiva. Rev Bras Enferm 2016;69(6):1154-63.

30. Novotny T, Hendrickson E, Soares ECC, Sereno AB, Kiene SM. HIV/AIDS, tuberculose e tabagismo no Brasil: uma sindemia que exige intervenções integradas. Cad Saúde Pública 2017;33Sup3:e00124215.

31. Halpern SC, Scherer JN, Roglio V, Faller S, Sordi A, Ornell F, et al. Vulnerabilidades clínicas e sociais em usuários de crack de acordo com a situação de moradia: um estudo multicêntrico de seis capitais brasileiras. Cad Saúde Pública 2017; 33(6):e00037517.

32. Soares JR, Donato M, Farias SNP de, Mauro MYC, Araujo EF dos S, Ghelman LG. Grupo focal como estrategia para a prevencao da recaida ao alcoolismo. Rev Enferm UERJ 2014;22(4):494-9.

33. Silveira CS, Passos PT, Soder TCH, Machado CPH, Fanfa LS, Carneiro M, et al. Perfil epidemiológico dos pacientes que abandonaram o tratamento para Tuberculose em um município prioritário do Rio Grande do Sul. Rev Epidemiol Control Infect 2012;2(2):4650.

34. Marjani M, Baghaei P, Malekmohammad M, Tabarsi P, Sharif-Kashani B, Behzadnia N, et al. Effect of pulmonary hypertension on outcome of pulmonary tuberculosis. Braz $\mathrm{J}$ Infect Dis 2014;18(5):487-90.

35. Durando P, Garbarino S, Orsi A, Alicino C, Dini G, Toletone A, et al. Prevalence and predictors of latent tuberculosis infection among Italian State Policemen engaged in assistance to migrants: a national cross-sectional study. BMJ Open 2016;6(10):e012011.

36. Silva NF, Faro A. Representações sociais acerca do futuro do usuário de crack: o que esperar? Psicol Saber Soc 2016;5(2):156-68.

37. Montoya ID. Treatment of patients with drug addiction and tuberculosis: new strategies for the future directions [Apresentação na Unidad de Investigación en Tuberculosis de Barcelona, 2014]. [citado 2017 Nov 8]. Disponível em: http://www.uitb.cat/wpcontent/uploads/2014/11/imontoya.pdf.

38. National Center for Chronic Disease Prevention and Health Promotion (US) Office on Smoking and Health. The health consequences of smoking - 50 years of progress a report of the surgeon general. Atlanta: Centers for Disease Control and Prevention (US); 2014.

39. Pereira JC, Silva MR, Costa RR, Guimarães MDC, Leite ICG. Profile and follow-up of patients with tuberculosis in a priority city in Brazil. Rev Saúde Pública 2015;49: 6.

40. Rabahi MF. Tuberculose e Tabagismo. Pulmão RJ 2012;21(1):46-9. 